\title{
Associations between Physical Activity and Submaximal Cardiorespiratory and Pulmonary Responses in Men
}

\author{
John C. Sieverdes*, Xuemei Sui and Steven N. Blair
}

University of South Carolina, Department of Exercise Science, Columbia, SC, USA

\begin{abstract}
Background: Habitual physical activity (PA) is associated with higher cardiorespiratory fitness values, but additional information is needed on the contributions of specific types and amounts of PA. Therefore the main aim of this study was to analyze the heart and lung function of a large cohort of men and compare these outcomes with
\end{abstract} various modes and volumes of PA.

Methods: We used data from 30,594 men from the Aerobics Center Longitudinal Study who were categorized into sedentary, swimmer, walker, and runner groups using self-report PA data collected during 1970-2005. Additional PA categories using MET-minutes/week were used to group men into 5 distinct levels of activity (0 MET-min, 1-499 MET-min, 500-999 MET-min, 1000-1499 MET-min, and $\geq 1500$ MET-min). Each participant also completed a maximal treadmill exercise test to quantify their fitness level. Cross-sectional analyses included general linear modeling and multiple comparisons adjusted for age, smoking status, and histories of myocardial infarction, stroke, diabetes, and hypertension.

Results: A dose-response linear effect was found for heart function variables across PA MET-min categories. Stronger associations for resting heart rate (HR), heart rate recovery (HRR), exercise HR, and exercise blood pressure were found with the runner and swimming groups when compared to the walkers and sedentary groups. Walkers had significantly better heart function than the sedentary group but only about half the effect seen in the swimmers and runners. Lung function findings showed greater absolute values in FVC and FEV1 across PA categories, but found no difference in lung function ratios (e.g FEV1/FVC\%).

Conclusions: We found beneficial linear associations with resting HR, exercise HR, HRR, fitness values, FVC, and FEV1 over increasing MET-min categories. This implies that habitual PA, such as walking, but especially swimming and running, when performed with adequate volume, are viable ways to gain benefits for heart health.

Keywords: Exercise; Pulmonary function; Cardiopulmonary; Cardiac Resp,onse

\section{Introduction}

Habitual leisure-time physical activity (PA) improves cardiorespiratory fitness (fitness) and is associated with reduced health outcomes such as cardiovascular disease and mortality [1,2]. Habitual PA has also been shown to lower resting heart rate (HR), improve heart rate recovery (HRR) [3], and lower resting blood pressure (BP) [4]. Heart function can be described as the various measures of efficiency for the heart and circulatory systems during rest and activity (e.g. resting and exercise $\mathrm{HR}$, resting and exercise BP, HRR, and fitness testing values). For the heart to reach its optimal potential, adequate pulmonary ventilation is also necessary, providing transportation of oxygen to the circulatory system where gas exchange takes place, though this is not the predominate limiting factor for exercise capacity in healthy individuals [5]. Capacity for ventilation can be described by lung function values from pulmonary testing (e.g. FEV1, FVC, FEV1/ FVC, FEV3, and PFR).

Several studies have investigated the prognostic value of $\mathrm{HR}$ response [6] and HRR to predict the risk of mortality [7,8]. Larger HRR values are evident in healthier populations and indicate proper reactivation of parasympathetic tone in the nervous system [8]. Regular PA has been shown to be protective against oxidative stress on the heart and vascular systems and is important for healthy functioning of the cardiovascular system [9]. Several studies have investigated the effects of habitual PA on heart and lung function and have shown higher rates of function for heart rate response [3,10,11], but not for lung function on average $[11,12]$. These studies focused on overall PA and have not investigated how specific activities or the volume of PA may influence heart or lung health. Experimental trials have studied the effects of short-term exercise on heart and lung function and found beneficial improvements [13]. However, limitations of such studies include smaller sample sizes, tight controls on exercise programs, were composed only of women, and were composed of limited types of exercises [13-15]. To the best of our knowledge, very few studies have investigated the effect of habitual exercise on submaximal BP and HR response in a healthy population [15]. Therefore, the aim of this study was to evaluate the association between heart and lung function at rest and during exercise using different modes of leisure-time PA along with categories of total PA volume during free-living conditions from a cohort of men.

\section{Methods \\ Participants}

The Aerobics Center Longitudinal Study (ACLS) is an American study of patients examined at the Cooper Clinic in Dallas, Texas, which started at the beginning of the 1970's. The Cooper Clinic is a preventative medical clinic where patients were self-referred or sent

*Corresponding author: John C. Sieverdes, MS, Doctoral Candidate, Department of Exercise Science, University of South Carolina, 921 Assembly St. Suite 212 Columbia, SC 29208, USA, Tel: 803-240-5790; Fax: 01 (803)777-2504; E-mail: sieverdj@email.sc.edu

Received September 22, 2011; Accepted November 18, 2011; Published November 22, 2011

Citation: Sieverdes JC, Sui X, Blair SN (2011) Associations between Physical Activity and Submaximal Cardiorespiratory and Pulmonary Responses in Men. J Sport Medic Doping Studie 1:102. doi:10.4172/2161-0673.1000102

Copyright: (c) 2011 Sieverdes JC, et al. This is an open-access article distributed under the terms of the Creative Commons Attribution License, which permits unrestricted use, distribution, and reproduction in any medium, provided the original author and source are credited. 
by a personal physician or employer for a medical examination. Participants underwent an extensive health screening, a thorough physical examination, and one counseling session for health, nutrition, and exercise between 1970 and 2005. The current study represents a cross-sectional analysis assessing lung and heart function variables during a preventive medical visit. We included 30,594 men in the analyses. Women were excluded in the current study because of the small sample size in the swimming category. Most of the men were Caucasian and from middle to upper socioeconomic strata. The Cooper Institute Institutional Review Board reviewed the study protocol annually.

\section{Clinical examination}

The ACLS examination required an overnight fast for participants' clinical visits before detailed blood analyses, BP, a graded exercise treadmill test, and standardized medical-history questionnaires were completed. Details of testing are described elsewhere [16]. Clinical assays were conducted in the clinical laboratory which met the quality control certifications of the Centers for Disease Control and Prevention lipid-standardization program. Serum samples for lipids and glucose were analyzed using standardized automated bioassays. Body-mass index (BMI, $\mathrm{kg} / \mathrm{m}^{2}$ ) was computed from measured height and weight. Hypertension was defined as any history of physician diagnosis of high BP, systolic BP of $140 \mathrm{~mm} \mathrm{Hg}$ or greater or diastolic BP of 90 or greater. Diabetes was defined from either a prior physician's diagnosis, if the participant used insulin, or if their fasting blood glucose was $\geq 126 \mathrm{mg} /$ dl. Smoking status was assessed in the medical-history questionnaire and was defined for analyses as current smokers, former smokers and never smokers. Alcohol use was defined in drinks per week, where one drink is equivalent to $12 \mathrm{oz}(3.41 \mathrm{dL})$ of beer, $5 \mathrm{oz}(1.421 \mathrm{dL})$ of wine, or $1.5 \mathrm{oz}(0.43 \mathrm{dL})$ of liquor. Histories of myocardial infarction, and stroke were from a medical history questionnaire.

\section{Lung function}

Lung function was defined by values from pulmonary ventilation testing. Lung function was assessed by trained laboratory staff using a Collins 421 Survey spirometer (Collins, MA) which is described elsewhere [17]. Forced expiratory volume in 1s (FEV1), forced vital capacity (FVC), Forced expiratory volume in 3 seconds (FEV3), and peak expiratory flow volume (PFR) were examined. All lung function variables, except FEV3, were expressed in Liters (L). Percentages were used for FEV3 and the ratios of FEV1 divided by FVC, where low lung function was identified if the ratio was $70 \%$ or lower.

\section{Heart function}

Heart function was defined here as the measures of efficiency for the heart and circulatory systems during rest and exercise (e.g. resting and exercise HR, resting and exercise BP, HRR, and fitness testing values). Cardiorespiratory fitness was assessed with a modified Balke protocol maximal treadmill test. Fitness was quantified by the maximum Metabolic equivalent $(\mathrm{MET})$ values $(1 \mathrm{MET}=3.5 \mathrm{ml} \mathrm{O} /$ $\mathrm{kg} / \mathrm{min}$, fitness $=(1.44 \times($ maximal minutes on treadmill $)+14.99) / 3.5)$ and treadmill test duration (in minutes). The test duration is strongly correlated to oxygen uptake in men $(r=0.94)$ [18]. The specifics of the treadmill test are described in other ACLS publications [19].

HR and HRR were measured using an electrocardiogram (ECG) before, during, and after the maximal exercise test. Resting HR values were measured after a 5 minute rest in the recumbent position. Heart rate was then measured at 5-minute intervals during the treadmill protocol. HRR was defined as the heart rate decline during the first 5 minutes after reaching maximal effort on the treadmill test (heart rate $_{\max }-$ heart rate ${ }_{5 \text { minutes of recovery }}$ ) [20] and taken during active recovery. Only HRR at 5 minutes was used because no other time points for HRR were measured during the tests. HR and HRR were continuous variables in this study.

BP was recorded using the first and fifth Korotkoff sounds with standard methodological procedures [21] after brief period of quiet restfulness. $\mathrm{BP}$ was taken at rest and every 5 minutes during the treadmill protocol

\section{Physical activity}

In the primary analysis, PA was categorized into four different activity groups (PA groups: sedentary, swimmers, walkers, runners) who self-reported their activity status during the prior 3 months $[22,23]$. Sedentary participants reported no leisure-time PA. Swimmers reported only swimming as their leisure-time activity. Walkers were identified as those who reported only walking as their activity and walked or walked for at least $75 \%$ of the time if they reported engaging in a run/walk/jog program. Runners were identified if they self reported running at a pace that was less than a $15 \mathrm{~min} / \mathrm{mile}$, or who walked less than $25 \%$ of their run/walk/jog program. Each participant could only be assigned to one group.

In the secondary analysis, a MET-minutes per week (MET-min) metric was used to categorize all men, regardless of the type of activity [24]. Use of the 2008 Physical Guidelines for Americans [25] was used to develop PA categories of 0 MET-min, 1-499 MET-min, 500-999 MET-min, 1000-1499 MET-min, and $\geq 1500$ MET-min. Time in each activity per week was multiplied by the value found in the compendium of physical activities [26] to develop the volume of activity per week in MET-min.

\section{Statistical analysis}

Cross-sectional analyses for the characteristics of men were presented by groups of leisure-time PA. Overall comparisons across groups were tested using general linear modeling for continuous variables and chi-square tests for categorical variables. Significance was tested between all leisure-time groups (swimmers, walkers, runners) against the sedentary group. Tukey-adjusted multiple comparisons were performed when comparing lung and heart function variables against the PA groups. Multiple comparisons included controlling for age, smoking status (current, former, never), history of myocardial infarction (yes, no), history of stroke (yes, no), history of hypertension (yes, no), history of diabetes (yes, no). Responses of heart and lung function over MET-min categories were depicted using the mean and 95\% CI from least squares analysis. These were also controlled for the variables in the tukey-adjusted modeling. All significance testing used a two-tailed test wih an $\alpha$ of $\mathrm{p}<0.05$. Statistical analysis was performed with SAS 9.2 (SAS Institute, Cary, N.C.)

\section{Results}

Characteristics of men across leisure-time PA groups (sedentary, swimmers, walkers, and runners) are shown in Table 1. Swimmers, walkers, and runners had better lipid profiles, had a lower BMI, and were less likely to smoke than sedentary participants. There were only 354 men in the swimming group, which comprised $1.2 \%$ of the study population.

\section{Heart and lung function across leisure-time PA groups}

Heart and lung function across sedentary and exercise PA groups are shown in (Table 2). Fewer men completed the exercise HR and BP measurements with 3052 sedentary men, 287 swimmers, 1045 walkers, 
Citation: Sieverdes JC, Sui X, Blair SN (2011) Associations between Physical Activity and Submaximal Cardiorespiratory and Pulmonary Responses in Men. J Sport Medic Doping Studie 1:102. doi:10.4172/2161-0673.1000102

Page 3 of 7

\begin{tabular}{|c|c|c|c|c|c|}
\hline \multicolumn{6}{|c|}{ Leisure-time physical activity groups } \\
\hline & Sedentary & Swimmers & Walkers & Runners & P-value \\
\hline $\mathrm{N}$ & 11229 & 354 & 2850 & 16161 & \\
\hline Age (mean $\pm \mathrm{SD}$, years) & $44.3 \pm 9.6$ & $46.6 \pm 10.4^{\mathrm{a}}$ & $46.0 \pm 10.4^{\mathrm{a}}$ & $44.3 \pm 9.6$ & $<0.0001$ \\
\hline Body mass index (mean $\pm \mathrm{SD}, \mathrm{kg} / \mathrm{m}^{2}$ ) & $27.2 \pm 4.2$ & $26.5 \pm 3.9^{\mathrm{a}}$ & $26.7 \pm 3.8^{\mathrm{a}}$ & $25.9 \pm 3.4^{\mathrm{a}}$ & $<0.0001$ \\
\hline \multicolumn{6}{|l|}{ Lipids (mean $\pm \mathrm{SD}, \mathrm{mmol} / \mathrm{L}$ ) } \\
\hline Total cholesterol & $215.0 \pm 41.2$ & $201.0 \pm 37.8^{a}$ & $209.6 \pm 40.1^{\mathrm{a}}$ & $205.2 \pm 40.6^{a}$ & $<0.0001$ \\
\hline HDL-C & $43.3 \pm 11.5$ & $48.0 \pm 11.8^{\mathrm{a}}$ & $45.1 \pm 11.7^{\mathrm{a}}$ & $47.6 \pm 12.3^{\mathrm{a}}$ & $<0.0001$ \\
\hline Triglycerides & $162.9 \pm 156.2$ & $124.3 \pm 79.6^{\mathrm{a}}$ & $142.7 \pm 114.5^{\mathrm{a}}$ & $122.6 \pm 88.2^{\mathrm{a}}$ & $<0.0001$ \\
\hline Fasting blood glucose (mean $\pm \mathrm{SD}, \mathrm{mmol} / \mathrm{L}$ ) & $102.3 \pm 21.9$ & $99.7 \pm 17.8$ & $101.2 \pm 19.1^{\mathrm{a}}$ & $99.3 \pm 14.3^{\mathrm{a}}$ & $<0.0001$ \\
\hline \multicolumn{6}{|l|}{ Blood pressure (mean SD, mmHg) } \\
\hline Systolic & $122.2 \pm 13.6$ & $122.8 \pm 12.6$ & $121.7 \pm 13.7$ & $121.5 \pm 13.7^{\mathrm{a}}$ & $<0.0001$ \\
\hline Diastolic & $82.1 \pm 9.7$ & $81.8 \pm 9.1$ & $81.5 \pm 9.7^{\mathrm{a}}$ & $80.6 \pm 9.5^{\mathrm{a}}$ & $<0.0001$ \\
\hline \multicolumn{6}{|l|}{ Smoking status } \\
\hline Former Smoker, \% & 30.0 & $16.7^{\mathrm{a}}$ & $32.9^{\mathrm{a}}$ & $35.4^{\mathrm{a}}$ & $<0.0001$ \\
\hline Current Smoker, \% & 28.4 & $14.4^{\mathrm{a}}$ & $16.3^{\mathrm{a}}$ & $12.1^{\mathrm{a}}$ & $<0.0001$ \\
\hline Alcohol consumption (mean $\pm \mathrm{SD}$, drinks/wk) & $4.6 \pm 7.5$ & $7.1 \pm 6.7^{\mathrm{a}}$ & $4.8 \pm 7.1$ & $5.1 \pm 6.7^{\mathrm{a}}$ & $<0.0001$ \\
\hline History of myocardial infarction, $\%$ & 1.2 & 0.3 & 1.5 & $1.7^{\mathrm{a}}$ & 0.005 \\
\hline History of stroke, \% & 0.2 & 0.0 & 0.2 & 0.2 & 0.69 \\
\hline History of hypertension, b, \% & 18.8 & 15.5 & $17.1^{\mathrm{a}}$ & $14.8^{\mathrm{a}}$ & $<0.0001$ \\
\hline History of Diabetes, c, \% & 4.4 & 5.4 & $3.3^{\mathrm{a}}$ & $3.4^{\mathrm{a}}$ & 0.0001 \\
\hline Low Lung Function FEV1/FVC <= 70\%, $\%$ & 13.6 & 10.7 & $12.3^{\mathrm{a}}$ & $12.1^{\mathrm{a}}$ & 0.003 \\
\hline
\end{tabular}

METs = maximal metabolic equivalents during the treadmill test; CVD = cardiovascular disease; HDL-C=high-density lipoprotein cholesterol; WJR = walker/jogger/runne a Statistical difference between sedentary compared to activity group at $p<0.05$ using least squares means

${ }^{b}$ Hypertension was defined as a systolic blood pressure $\geq 140 \mathrm{~mm} \mathrm{Hg}$ or diastolic blood pressure $\geq 90 \mathrm{~mm} \mathrm{Hg}$ or a history of physician diagnosis or taking medication for hypertension

' Diabetes mellitus was defined as a fasting plasma glucose concentration $\geq 6.993 \mathrm{mmol} / \mathrm{L}$, a history of physician diagnosis, or insulin use

$P$-value denotes overall significant differences for at least one group

Table 1: Characteristics across physical activity groups.

\begin{tabular}{|c|c|c|c|c|c|}
\hline & Sedentary & Swimmers & Walkers & Runners & P-value \\
\hline Lung Function & $N=11229$ & $N=354$ & $N=2850$ & $N=16161$ & \\
\hline FEV1, L & $3.68(0.70)^{a b c}$ & $3.94(0.75)^{\mathrm{ad}}$ & $3.79(0.69)^{\text {bde }}$ & $3.92(0.67)^{\mathrm{ce}}$ & $<0.0001$ \\
\hline FVC, L & $4.73(0.82)^{\mathrm{abc}}$ & $5.01(0.89)^{\mathrm{ad}}$ & $4.85(0.82)^{\text {bde }}$ & $5.01(0.79)^{\mathrm{ce}}$ & $<0.0001$ \\
\hline FEV1/FVC, \% & $78.00(7.54)^{\mathrm{a}}$ & $78.66(6.60)$ & $78.25(7.28)$ & $78.38(7.18)^{\mathrm{a}}$ & 0.19 \\
\hline FEV3, \% & $91.94(7.40)^{\mathrm{a}}$ & $91.40(14.66)$ & $93.26(25.88)$ & $93.45(20.83)^{a}$ & 0.006 \\
\hline PFR, L/min & $8.67(2.71)^{\mathrm{ab}}$ & $8.82(2.11)$ & $8.84(2.22)^{\mathrm{ac}}$ & $9.17(2.23)^{\mathrm{bc}}$ & $<0.0001$ \\
\hline \multicolumn{6}{|l|}{ Heart Function } \\
\hline Resting Heart Rate & $64.6(10.5)^{\mathrm{abc}}$ & $57.5(9.0)^{\mathrm{ad}}$ & $61.4(10.3)^{\text {bde }}$ & $57.5(10.2)^{\mathrm{ce}}$ & $<0.0001$ \\
\hline Exercise heart rate at $5 \mathrm{~min}$ & $117.0(15.6)^{\mathrm{abc}}$ & $106.8(14.8)^{\mathrm{ad}}$ & $111.0(14.2)^{\text {bde }}$ & $105.7(14.5)^{\mathrm{ce}}$ & $<0.0001$ \\
\hline Exercise heart rate at $10 \mathrm{~min}$ & $142.9(17.0)^{\mathrm{abc}}$ & $131.6(17.2)^{\mathrm{ad}}$ & $136.7(16.5)^{\text {bde }}$ & $129.7(16.8)^{\mathrm{ce}}$ & $<0.0001$ \\
\hline Exercise heart rate at $15 \mathrm{~min}$ & $166.0(16.1)^{\mathrm{abc}}$ & $156.2(16.8)^{\mathrm{ad}}$ & $160.7(16.1)^{\text {bde }}$ & $153.3(16.9)^{\mathrm{ce}}$ & $<0.0001$ \\
\hline Exercise systolic BP at $5 \mathrm{~min}$ & $153.9(22.8)^{\mathrm{abc}}$ & $147.3(20.1)^{a}$ & $150.6(21.8)^{\mathrm{bd}}$ & $148.5(20.5)^{\text {cd }}$ & $<0.0001$ \\
\hline Exercise systolic BP at $10 \mathrm{~min}$ & $172.8(24.2)^{\mathrm{abc}}$ & $166.4(22.0)^{\mathrm{ad}}$ & $170.5(24.2)^{\text {bde }}$ & $166.8(22.6)^{\mathrm{ce}}$ & $<0.0001$ \\
\hline Exercise systolic BP at $15 \mathrm{~min}$ & $181.7(21.9)$ & $181.1(22.1)$ & $182.1(22.3)$ & $181.0(22.2)$ & 0.29 \\
\hline Heart rate recovery & $66.9(15.3)^{\mathrm{abc}}$ & $64.7(14.0)^{\text {ade }}$ & $68.5(15.1)^{\text {bdf }}$ & $72.74(14.0)^{\text {cef }}$ & $<0.0001$ \\
\hline \multicolumn{6}{|l|}{ Exercise Capacity and Volume } \\
\hline Exercise tolerance (mean $\pm \mathrm{SD}, \mathrm{METs}$ ) & $10.13(1.95)^{\mathrm{abc}}$ & $11.95(2.31)^{\text {ade }}$ & $11.12(2.10)^{\text {bdf }}$ & $12.76(2.41)^{\text {cef }}$ & $<0.0001$ \\
\hline Treadmill test duration (mean $\pm \mathrm{SD}$, minutes) & $14.5(4.1)^{\mathrm{abc}}$ & $18.6(4.7)^{\text {ade }}$ & $16.9(4.4)^{\text {bdf }}$ & $20.2(4.8)^{\text {cef }}$ & $<0.0001$ \\
\hline MET-minutes (mean \pm SD, MET-min/wk) & $0(0)^{\mathrm{abc}}$ & $1398.7(1444.8)^{\mathrm{ad}}$ & $490.8(841.8)^{\text {bde }}$ & $1267.0(1345.7)^{\mathrm{ce}}$ & $<0.0001$ \\
\hline
\end{tabular}

FEV1, forced expiratory volume in first second; FEV3, forced expiratory volume in three seconds; FVC, forced vital capacity; PRF, peak expiratory flow volume; MET, metabolic unit

a,b,c,d,e,f pairs denotes significant differences comparing each physical activity group with one another at $p<0.05$ using multiple comparisons adjusted by Tukey's post hoc test

All comparisons, except MET-minutes were adjusted for age, smoking status (never, former, current), history of myocardial infarction (yes, no), history of stroke (yes, no), history of hypertension (yes, no), history of diabetes (yes, no)

$\mathrm{P}$-value denotes overall significant differences for at least one group

Table 2: Heart and lung function characteristics of men. 
and 5876 runners reaching the 5-minute HR stage. The percentage of 10 - and 15 - minute completers for HR were $90 \%$ and $51 \%$ for sedentary men, $95 \%$ and $76 \%$ for swimmers, $94 \%$ and $64 \%$ for walkers, and $98 \%$ and $85 \%$ for runners. The numbers completing each stage for BP were approximately equal to the sample size for HR at each stage. Multiple comparisons for heart function showed that swimmers and runners were similar in respect to resting and exercise HR. Both showed lower values than the sedentary and walker groups for each exercise time during the treadmill protocol and did not differ from each other. Walkers also showed significantly lower values than the sedentary group for HR response.

All exercise groups had lower responses to BP at 5- and 10minute time intervals during the treadmill protocol when compared to the sedentary group. At 5 minutes, swimmers had a BP that was no different than the other groups, but runners had lower BP than the walkers. At 10 minutes, there was no difference between the swimmers and runners, but both were significantly lower than the walkers. At 15 minutes, no differences were found among all four groups, which could be attributed to the reduced number of un-fit men in the sedentary group that made it to 15 minutes.

Runners had the largest change in HRR followed by the walkers, sedentary, and finally swimmers. All groups showed that HRR was significantly different from one another.

All PA groups resulted in significant differences for treadmill test duration. Treadmill time was converted to MET values, which ranged from 10.13 for the sedentary group to 12.76 for the runners. MET-min are shown for each group with walkers obtaining an average of 490 MET-min. The swimmers and runners each averaged more than twice the walkers' values, while all PA groups had high standard deviations.

Multiple comparisons for lung function showed significantly higher values of FEV1 and FVC for all active groups compared to the sedentary group. Although all PA groups had higher values for FEV1/ FVC\%, the only statistical difference was between the sedentary and runner groups. Runners also had a higher mean values for FEV3\%. For PFR, the sedentary group had lower values than the walkers and runners, though no difference was seen when compared to the runners.

\section{Heart and lung function across PA categories (MET-min)}

(Figures 1-3) show the secondary analyses using five categories of MET-min to investigate trends in lung and heart function. The volume of activity for each PA category were as follows (in MET-min, mean (standard deviation)): 0(0) for category 1, 255(136) for category $2,737(142)$ for category $3,1227(147)$ for category 4 , and 2738(1625) for category 5. Heart function is illustrated in (Figure 1). HR at each 5-minute treadmill test duration showed an inverse trend across the incremental categories of PA. A 20 beats per minute (bpm) difference between the lowest and highest PA category was observed. HRR also trended upward while resting HR trended downward across PA categories. BP trends were found at 5- and 10-minutes test durations indicating a dose-response association, but this was not evident at 15 minutes or at rest. Unlike the HR which showed a linear curve, BP response plateaued at the 500-999 MET-minute/week category. Lastly, Figure 2 illustrates a linear increase for fitness variables with increasing levels of PA categories.

Lung function is illustrated in Figure 3. An upward trend was seen in FVC and FEV1 across increasing levels of PA, indicating higher absolute lung capacity. The slopes of FEV3 and FEV1/FVC\% were relatively flat, indicating no increase in lung function across higher PA categories.

\section{Discussion}

The main finding of the study was the dose-response association of heart function across PA categories (e.g. across increasing levels
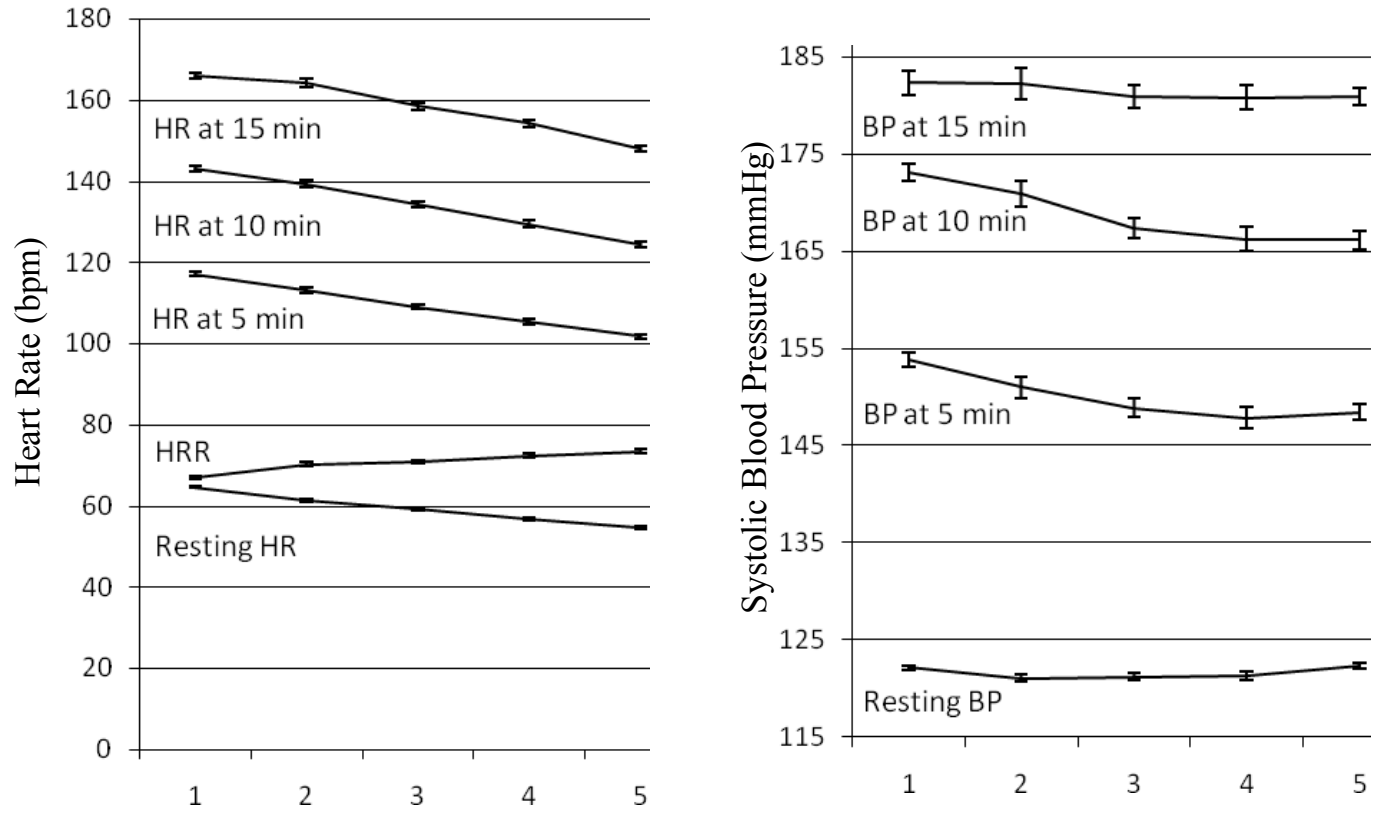

Physical Activity Categories

Figure 1: Response of Heart Rate and Blood Pressure over Five Categories of Physical Activity. Mean and $95 \% \mathrm{Cl}$ of the mean using least squares mean. Model was adjusted for by age, smoking status, history of heart attack, history of stroke, history of hypertension, and history of diabetes. Physical activity categories represent $(1=$ 0 MET-minutes; 2= 1-499 MET-minutes; 3= 500-999 MET-minutes; 4= 1000-1499 MET-minutes; 5 = 1500 MET-minutes or greater). 
of MET-min categories) in a large sample of men. The stronger associations found in the runner and swimming groups with resting $\mathrm{HR}$, exercise HR, and exercise BP may be attributable to the volume of the exercise rather than the type. Similar patterns were found with increases in treadmill duration time and their respective maximal METs showing a linear increase across PA categories. Lung function findings showed greater absolute values in FVC and FEV1 for increasing PA categories, but changes in lung function ratios were negligible.

The higher intensity activities such as swimming and running were found to have larger positive associations with heart function. These two activities, which are usually vigorous in nature, were associated with lower resting HR and lower exercise HR and BP. The differences between swimmers and runners may be attributed to the specificity of the treadmill test, which is better suited for runners. Walkers also showed statistically healthier heart function values when compared to the sedentary group, though the association was not as strong as the runners and swimmers. In fact, walkers had approximately $50 \%$ of the benefit the runners and swimmers groups experienced when each were compared to the sedentary group. Due to the cross-sectional methodology, this study could not examine whether the intensity of the PA groups or the volume of MET-minutes from the PA categories was the determinant factor for better heart functioning. When investigating $\mathrm{HR}$ and $\mathrm{BP}$ response to exercise among the PA groups, we noticed a plateau effect on BP. Further analysis using only men who reached the 15-minute time point of the treadmill test were re-examined and compared to (Figure 1). In this subsample, BP response at 5-, 10-, and 15- minute increments was flat across PA categories (mean values of $\mathrm{BP}$ in mmHg: 119.9-122.5 (5-minutes), 144.4-146.5 (10-minutes), 180.8-181.9 (15-minutes)). For HR response, an attenuated but evident slope was still seen across the PA categories similar to (Figure 1) with a range between 10.3 to $18.1 \mathrm{bpm}$ difference between the highest and lowest category (e.g. 10 -minutes response $1^{\text {st }} \mathrm{PA}$ category $\mathrm{HR}=137.3$ to $5^{\text {th }} \mathrm{PA}$ group $\left.\mathrm{HR}=123.15\right)$. This in effect controls for fitness and indicates that PA has a stronger association on HR response than on $\mathrm{BP}$ where higher fit men regardless of PA category had lower BP values.

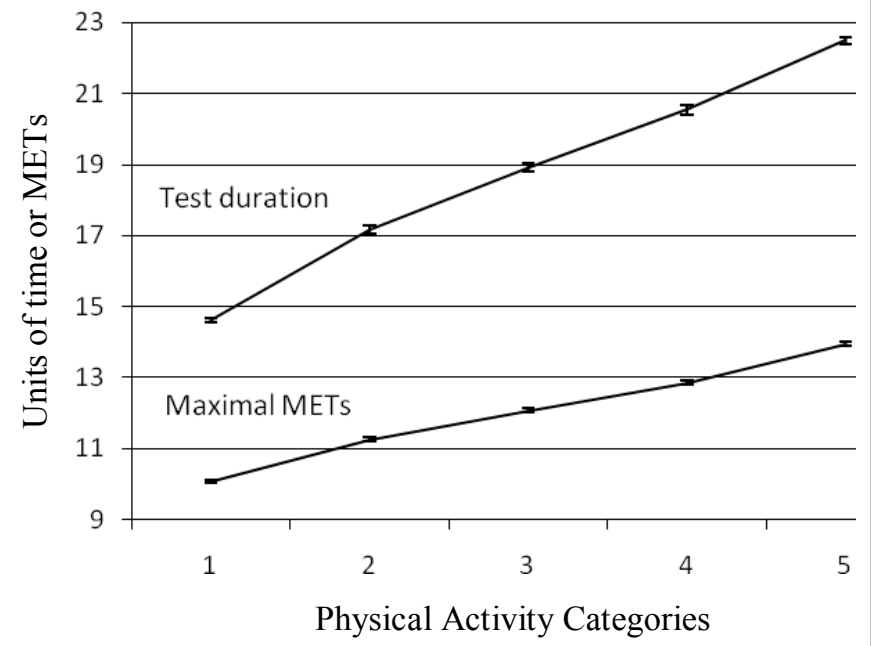

Figure 2: Fitness testing and maximal METs attained during the Modified Balke treadmill test across five physical activity categories. Mean and $95 \% \mathrm{Cl}$ of the mean using least squares mean. Model was adjusted for by age, smoking status, history of heart attack, history of stroke, history of hypertension, and history of diabetes. Physical activity categories represent (1= 0 MET-minutes; 2= 1-499 MET-minutes; 3= 500-999 MET-minutes; 4= 1000-1499 MET-minutes; 5 = 1500 MET-minutes or greater).
Our results are similar to previous studies that have examined the effect of resting heart and lung function with exercise. Cotes et al. showed that sub-maximal HR decreases as habitual exercise levels increase. The current study showed the same outcome but with the addition of BP response and over a wider range of activities including a sedentary comparison group. Murray and colleagues reported a reduction in resting HR and systolic BP after 2 weeks of aerobic training [4]. We also observed differences between the sedentary and active groups for resting HR, but not for BP. HRR has been shown to be associated with fitness status where trained subjects' $\mathrm{HR}$, regardless of age, recover faster than untrained subjects [3]. This represents proper autonomic heart function, even at the five minutes time-point used in this analysis. Other studies examine the HRR at one or two minutes, though we did not have the data available at those time points postexercise test to compare [3,7]. We saw similar results for increased HRR for more active groups when compared to these studies. For lung function, Miller and colleagues studied the PA and lung function of several populations and found habitual activity, environmental temperature, and body muscle to be more important predictors of fitness [11]. In this ACLS study, we found similar findings across the PA categories (Figure 3), though like Miller et al., found some large absolute differences between groups.

Some of the most notable changes in heart function are the result of exercise adaptations to the cardiovascular system. Of these, the maintenance or increase in left ventricular (LV) mass, increases in the LV ejection fraction, increases in stroke volume, and reduction of oxidative stress [9] could explain the reduction of resting and exercise HR in the PA groups. Moving larger volumes of blood with each beat reduces the demand of the heart, thus reducing resting and exercise HR. Vascular effects of exercise include reductions of aortic stiffness, increases in endothelial vasodilation, and increases in systemic and venular capillaries [9], which could result in reduced BP during exercise. The increases in HRR are suggestive that higher volumes of PA improve parasympathetic activity and faster introduction of vagal tone [27]. No effects of habitual exercise training on lung function variables for healthy populations has been found since total lung volume cannot be changed [11]. Most lung function changes with exercise training is evident with alveolar ventilation and alveolar to arterial oxygen and carbon dioxide exchange rather than ventilation flow dynamics or changes to pressure-generating capabilities of the respiratory muscles [28].

Our main limitation in this study is the cross-sectional methodology, therefore casual inferences from the PA groups or categories cannot be made. Another limitation involves the standardization of the treadmill testing across all patients which may not provide an accurate fitness assessment for the swimmers due to the specificity of the exercise. Lastly, the study population represented men from relatively high socioeconomic strata that included few minorities, which limits the generalizability of the findings. With these limitations are a few notable strengths. The ACLS is a large database that has numerous clinical measures to allow comparisons of pulmonary and heart function. The extensive detail resulting from the PA questionnaire allowed the categorization of swimmers and the creation of MET-minutes, which is seldom used for comparisons in large studies.

\section{Conclusions}

In this study, we found healthier heart function in men who were physically active than those who led a sedentary lifestyle. Swimming, running, or performing a combination of activities that resulted in 1500 or more MET-minutes of PA had the largest benefits. The associations 
Citation: Sieverdes JC, Sui X, Blair SN (2011) Associations between Physical Activity and Submaximal Cardiorespiratory and Pulmonary Responses in Men. J Sport Medic Doping Studie 1:102. doi:10.4172/2161-0673.1000102
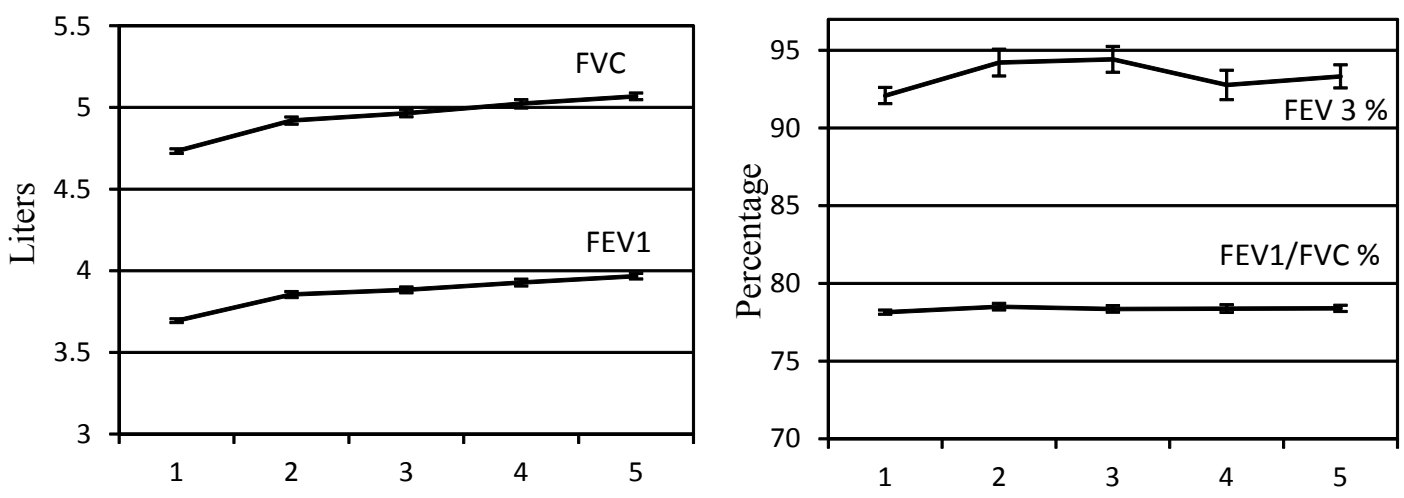

Phvsical Activitv Categories

Figure 3: Lung response by over five levels of physical activity categories. Mean and $95 \% \mathrm{Cl}$ of the mean using least squares mean. Model was adjusted for by age, smoking status, history of heart attack, history of stroke, history of hypertension, and history of diabetes. Physical activity categories represent $(1=0 \mathrm{MET}-\mathrm{minutes} ; 2=$ 1-499 MET-minutes; 3= 500-999 MET-minutes; 4= 1000-1499 MET-minutes; 5 = 1500 MET-minutes or greater)

with PA categories showed a linear effect on resting HR, exercise HR, fitness, HRR, and a plateau effect on exercise BP. When compared to the sedentary group, walkers had approximately half the associated functional benefit when compared to more vigorous activities such as swimming and running. This shows the added benefit of choosing a more vigorous activity to stay healthy. Future comparisons should control for the volume of activity to study the effects of the intensity of habitual PA on heart function.

\section{Acknowledgements}

The authors thank the Cooper Clinic physicians and technicians for collecting the data, and staff at the Cooper Institute for data entry and data management.

\section{Disclosure Statement of Funding}

Funding for this research was supported by a grant from the National Swimming Pool Foundation, and National Institutes of Health grants AG06945, HL62508, and DK088195. None of the sponsors had any role in the creation of this paper.

\section{References}

1. Haskell WL, Lee IM, Pate RR, Powell KE, Blair SN, et al. (2007) Physica activity and public health: updated recommendation for adults from the American College of Sports Medicine and the American Heart Association. Med Sci Sports Exerc 39: 1423-1434.

2. Kesaniemi YK, Danforth E Jr, Jensen MD, Kopelman PG, Lefebvre P, et al. (2001) Dose-response issues concerning physical activity and health: an evidence-based symposium. Med Sci Sports Exerc 33: S351-S358.

3. Darr KC, Bassett DR, Morgan BJ, Thomas DP (1988) Effects of age and training status on heart rate recovery after peak exercise. Am J Physiol 254: H340-343.

4. Murray A, Delaney T, Bell C (2006) Rapid onset and offset of circulatory adaptations to exercise training in men. J Hum Hypertens 20: 193-200.

5. Rowe SA, Zahka KG, Manolio TA, Horneffer PJ, Kidd L (1991) Lung function and pulmonary regurgitation limit exercise capacity in postoperative tetralogy of Fallot. J Am Coll Cardiol 17: 461-466.

6. Leeper NJ, Dewey FE, Ashley EA, Sandri M, Tan SY, et al. (2007) Prognostic value of heart rate increase at onset of exercise testing. Circulation 115: 468474

7. Cole CR, Foody JM, Blackstone EH, Lauer MS (2000) Heart rate recovery after submaximal exercise testing as a predictor of mortality in a cardiovascularly healthy cohort. Ann Intern Med $132: 552-555$.

8. Cole CR, Blackstone EH, Pashkow FJ, Snader CE, Lauer MS (1999) Heart-rate recovery immediately after exercise as a predictor of mortality. $\mathrm{N}$ Engl $\mathrm{J}$ Med 341: 1351-1357.

9. Gielen S, Schuler G, Adams V (2010) Cardiovascular effects of exercise training: molecular mechanisms. Circulation 122: 1221-1238.
10. Cotes JE, Dabbs JM, Daley C, Hall AM, Johnson GR, et al. (1971) Aboveaverage exercise capacity in competition cyclists; relationship to body muscle J Physiol 218: 63P-64P.

11. Miller GJ, Cotes JE, Hall AM, Salvosa CB, Ashworth A (1972) Lung function and exercise performance of healthy Caribbean men and women of African ethnic origin. Q J Exp Physiol Cogn Med Sci 57: 325-341.

12. Dempsey JA, Gledhill N, Reddan WG, Forster HV, Hanson PG, et al. (1977) Pulmonary adaptation to exercise: effects of exercise type and duration, chronic hypoxia and physical training. Ann N Y Acad Sci 301: 243-261.

13. Church TS, Earnest CP, Skinner JS, Blair SN (2007) Effects of different doses of physical activity on cardiorespiratory fitness among sedentary, overweight or obese postmenopausal women with elevated blood pressure: a randomized controlled trial. JAMA 297: 2081-2091.

14. Arsenault BJ, Cote M, Cartier A, Lemieux I, Despres JP, et al. (2009) Effect of exercise training on cardiometabolic risk markers among sedentary, but metabolically healthy overweight or obese post-menopausal women with elevated blood pressure. Atherosclerosis 207: 530-533.

15. Ekblom B, Astrand PO, Saltin B, Stenberg J, Wallstrom B (1968) Effect of training on circulatory response to exercise. J Appl Physiol 24: 518-528.

16. Blair SN, Kampert JB, Kohl HW, Barlow CE, Macera CA, et al. (1996) Influences of cardiorespiratory fitness and other precursors on cardiovascular disease and all-cause mortality in men and women. JAMA 276: 205-210.

17. Cheng YJ, Macera CA, Addy CL, Sy FS, Wieland D, et al. (2003) Effects of physical activity on exercise tests and respiratory function. $\mathrm{Br} \mathrm{J}$ Sports Med 37: 521-528.

18. Pollock ML, Bohannon RL, Cooper KH, Ayres JJ, Ward A, et al. (1976) A comparative analysis of four protocols for maximal treadmill stress testing. Am Heart J 92: 39-46.

19. Blair SN, Kohl HW, Paffenbarger RS Jr, Clark DG, Cooper KH, et al. (1989) Physical fitness and all-cause mortality. A prospective study of healthy men and women. JAMA 262: 2395-2401.

20. Cheng YJ, Lauer MS, Earnest CP, Church TS, Kampert JB, et al. (2003) Heart rate recovery following maximal exercise testing as a predictor of cardiovascular disease and all-cause mortality in men with diabetes. Diabetes Care 26: 2052-2057.

21. Pickering TG, Hall JE, Appel LJ, Falkner BE, Graves J, et al. (2005) Recommendations for blood pressure measurement in humans and experimental animals: Part 1: blood pressure measurement in humans: a statement for professionals from the Subcommittee of Professional and Public Education of the American Heart Association Council on High Blood Pressure Research. Hypertension 45: 142-161.

22. Chase NL, Sui X, Blair SN (2008) Comparison of the Health Aspects of Swimming with Other Types of Physical Activity and Sedentary Lifestyle Habits. International Journal of Aquatic Research and Education 2: 151-161. 
Citation: Sieverdes JC, Sui X, Blair SN (2011) Associations between Physical Activity and Submaximal Cardiorespiratory and Pulmonary Responses in Men. J Sport Medic Doping Studie 1:102. doi:10.4172/2161-0673.1000102

Page 7 of 7

23. Sieverdes JC, Ray BM, Sui X, Lee DC, Hand GA, et al. (2011) Association between Leisure-Time Physical Activity and Depressive Symptoms in Men. Med Sci Sports Exerc In Press.

24. Lee DC, Sui X, Ortega FB, Kim YS, Church TS, et al. (2011) Comparisons of leisure-time physical activity and cardiorespiratory fitness as predictors of allcause mortality in men and women. Br J Sports Med 45: 504-510.

25. US Department of Health and Human Services (2008) physical activity guidelines for Americans
26. Ainsworth BE, Haskell WL, Whitt MC, Irwin ML, Swartz AM, et al. (2000) Compendium of physical activities: an update of activity codes and MET intensities. Med Sci Sports Exerc 32: S498-S504.

27. Imai K, Sato H, Hori M, Kusuoka H, Ozaki H, et al. (1994) Vagally mediated heart rate recovery after exercise is accelerated in athletes but blunted in patients with chronic heart failure. J Am Coll Cardiol 24: 1529-1535.

28. Dempsey JA, Johnson BD, Saupe KW (1990) Adaptations and limitations in the pulmonary system during exercise. Chest $97: 81 \mathrm{~S}-87 \mathrm{~S}$. 